\title{
Subdoses of 17DD yellow fever vaccine elicit equivalent virological/immunological kinetics timeline
}

\author{
Ana Carolina Campi-Azevedo ${ }^{1 *}{ }^{*}$, Paula de Almeida Estevam ${ }^{1 \dagger}$, Jordana Grazziela Coelho-dos-Reis ${ }^{1}$, \\ Vanessa Peruhype-Magalhães ${ }^{1}$, Gabriela Villela-Rezende ${ }^{1}$, Patrícia Flávia Quaresma ${ }^{1}$, Maria de Lourdes Sousa Maia², \\ Roberto Henrique Guedes Farias², Luiz Antonio Bastos Camacho³, Marcos da Silva Freire², Ricardo Galler², \\ Anna Maya Yoshida Yamamura², Luiz Fernando Carvalho Almeida², Sheila Maria Barbosa Lima², \\ Rita Maria Ribeiro Nogueira ${ }^{4}$, Gloria Regina Silva Sá2, Darcy Akemi Hokama², Ricardo de Carvalho², \\ Ricardo Aguiar Villanova Freire ${ }^{5}$, Edson Pereira Filho ${ }^{5}$, Maria da Luz Fernandes Leal ${ }^{2}$, Akira Homma², \\ Andréa Teixeira-Carvalho ${ }^{1}$, Reinaldo Menezes Martins ${ }^{2}$ and Olindo Assis Martins-Filho ${ }^{1}$
}

\begin{abstract}
Background: The live attenuated 17DD Yellow Fever vaccine is one of the most successful prophylactic interventions for controlling disease expansion ever designed and utilized in larger scale. However, increase on worldwide vaccine demands and manufacturing restrictions urge for more detailed dose sparing studies.

The establishment of complementary biomarkers in addition to PRNT and Viremia could support a secure decision-making regarding the use of 17DD YF vaccine subdoses. The present work aimed at comparing the serum chemokine and cytokine kinetics triggered by five subdoses of 17DD YF Vaccine.

Methods: Neutralizing antibody titers, viremia, cytokines and chemokines were tested on blood samples obtained from eligible primary vaccinees.

Results and discussion: The results demonstrated that a fifty-fold lower dose of 17DD-YF vaccine (587 IU) is able to trigger similar immunogenicity, as evidenced by significant titers of anti-YF PRNT. However, only subdoses as low as 3,013 IU elicit viremia kinetics with an early peak at five days after primary vaccination equivalent to the current dose $(27,476 \mathrm{IU})$, while other subdoses show a distinct, lower in magnitude and later peak at day 6 post-vaccination. Although the subdose of $587 \mathrm{IU}$ is able to trigger equivalent kinetics of IL-8/CXCL-8 and MCP-1/CCL-2, only the subdose of $3,013 \mathrm{IU}$ is able to trigger similar kinetics of MIG/CXCL-9, pro-inflammatory (TNF, IFN- $\gamma$ and IL-2) and modulatory cytokines (IL-5 and IL-10).
\end{abstract}

Conclusions: The analysis of serum biomarkers IFN- $\gamma$ and IL-10, in association to PRNT and viremia, support the recommendation of use of a ten-fold lower subdose (3,013 IU) of 17DD-YF vaccine.

Keywords: Yellow fever, Vaccine, Dose-response, Viremia, Cytokines, Ckemokines

\footnotetext{
* Correspondence: accampi@cpqrr.fiocruz.br

${ }^{\dagger}$ Equal contributors

'Centro de Pesquisas René Rachou - Fiocruz, Belo Horizonte, Minas Gerais,

Brazil

Full list of author information is available at the end of the article
} 


\section{Background}

The yellow fever (YF) virus is a mosquito-borne flavivirus that causes hemorrhagic disease with jaundice in people inhabiting tropical areas [1-3]. The incidence of YF dropped significantly after the development of live attenuated vaccines in the 1930s [3]. The YF vaccines (17D and 17DD) are one of the most successful prophylactic interventions for controlling disease expansion ever designed and utilized in larger scale [4].

Recent evidences for expansion of viral circulation in tropical areas estimate that over 900 million people are at risk of infection [5]. It is now effective that the YF vaccine is been currently used to protect travelers and is incorporated in childhood vaccination programs in many countries, with millions of doses distributed annually around the globe. Up to present, there are 6 producers of yellow fever vaccines, but only 4 are prequalified by the World Health Organization and supply vaccines to international agencies: Bio-Manguinhos (Brazil), SanofiPasteur (France), Institute Pasteur in Dakar (Senegal) and Chumakov Institute - Institute of Poliomyelitis and viral encephalitides (Russia Federation) [6]. In 2008, the sudden increase on the demand for YF vaccine forced Bio-Manguinhos, the Brazilian supplier of 17DD vaccine, to interrupt any vaccine exporting to other countries [7]. This sudden rise in vaccine demand was due to the large epidemic of yellow fever in tropical South America, caused by the increase of Aedes aegypti infestation levels in many urban cities, in addition to the frequent movement of susceptible individuals from yellow fever-free to endemic areas [7].

Thus, the spreading of risk areas and the restricted group of YF vaccine manufacturers creates a shortage on YF vaccine supply worldwide, which urges for new strategies of vaccination protocols including validation of new seed lots, need and timing of booster doses to maintain long lasting protection as well as dose sparing studies [8]. In regards to dose, the minimal number of viral particles has been established by $\mathrm{WHO}$ as at least $5,000 \mathrm{PFU}$ or approximately 3,000 IU. However, the maximum dose has not been established [5,9]. Previous studies have reported that the number of virions in the 17DD-YF vaccine produced by Bio-Manguinhos/FIOCRUZ is on average approximately seven times higher (2.3 to 12.0 times) than the minimal dose established by WHO $[5,9]$. The fine-tuning of the vaccine dose in current use to lower number of viral particles, above the minimum required by WHO, could increase the vaccine availability and supply the worldwide increasing needs. However, it is important to guarantee that lower doses are able to induce similar protection [9]. It has been proposed by Lopes et al. [10] that doses higher than 200 PFU (approximately $100 \mathrm{IU})$ were able to induce $100 \%$ of seroconversion. However, recent evidence has shown that doses as low as 47 times $(1,122 \mathrm{PFU}$ or $587 \mathrm{IU})$ the reference are required to induce equivalent seroconversion rates [5,9]. It is clear that a better understanding of the virological/immunological features upon YF subdoses vaccination is relevant to further support changes in the minimal dose recommended by the YF-vaccination guidelines. Therefore, in the present study, individuals who had primary vaccination with subdoses of the 17DD-YF vaccine were tested for virological/immunological serum biomarkers, such as the viral load, chemokines and cytokines as well as neutralizing antibody titers. The kinectics of such biomarkers, taken in association, highly advice for alternative and equivalent vaccination protocols with subdoses of the 17DD-YF vaccine.

\section{Methods}

\section{Design of the study}

The present study was performed by the Collaborative Group for Studies of Yellow Fever Vaccine aiming to investigate virological and immunological features induced by subdoses of the 17DD-YF Vaccine after approval of the Ethical Committee for studies with human subjects (CPqRR/FIOCRUZ \#22/2010). The study population consisted of 900 healthy, adult (age average 19.4 years), army, male conscripts from Rio de Janeiro enrolled in a screening phase. All participants informed not being vaccinated for Yellow Fever previously and agreed with and signed a written consent form. Participants were distributed randomly into six study groups (150 subject/group) each of which were given different the currently used dose of 17DD-YF vaccine (27,476 IU 52,480 PFU) and five alternative formulation with decreasing number of viral particles (10,447 IU - 19,953PFU; 3,013 IU - 5,754PFU; 587 IU - 1,122PFU; 158 IU 302PFU and $31 \mathrm{IU}$ - 59PFU), as shown in Figure 1. Excluding criteria were: 1) missing blood collection at Baseline $(\mathrm{n}=50), 2)$ insufficient serum sample volume $(\mathrm{n}=147), 3)$ Seropositivity (PRNT $\left.\geq 2.70 \log _{10} \mathrm{mIU} / \mathrm{mL}\right)$ at Baseline $(\mathrm{n}=75)$ or 4$)$ timeline interval of blood collection $>34$ days $(n=37)$. The eligible population $(n=590)$ was selected for pairing with baseline sample according to the number of blood samples available (two blood samples, $\mathrm{n}=295 \rightarrow 295$ pairs and three blood samples, $\mathrm{n}=295 \rightarrow 590$ pairs), resulting in a total of 885 paired samples. Paired samples were grouped according to dose given and referred as 27,476 IU ( $\mathrm{n}=157), 10,447 \mathrm{IU}$ ( $\mathrm{n}=144), 3,013 \mathrm{IU}(\mathrm{n}=150), 587$ IU $(\mathrm{n}=140), 158 \mathrm{IU}$ $(\mathrm{n}=145)$ and $31 \mathrm{IU}(\mathrm{n}=149)$. The experimental design consisted of eight timepoints: before (Baseline) and days after primary vaccination (D3, D4, D5, D6, D7, D15 and D30). Each timepoint was comprised in average of 21 paired samples for each dose. The Plaque Reduction Neutralization Test (PRNT) was performed at baseline and D30. Viremia was assayed at D3, D4, 


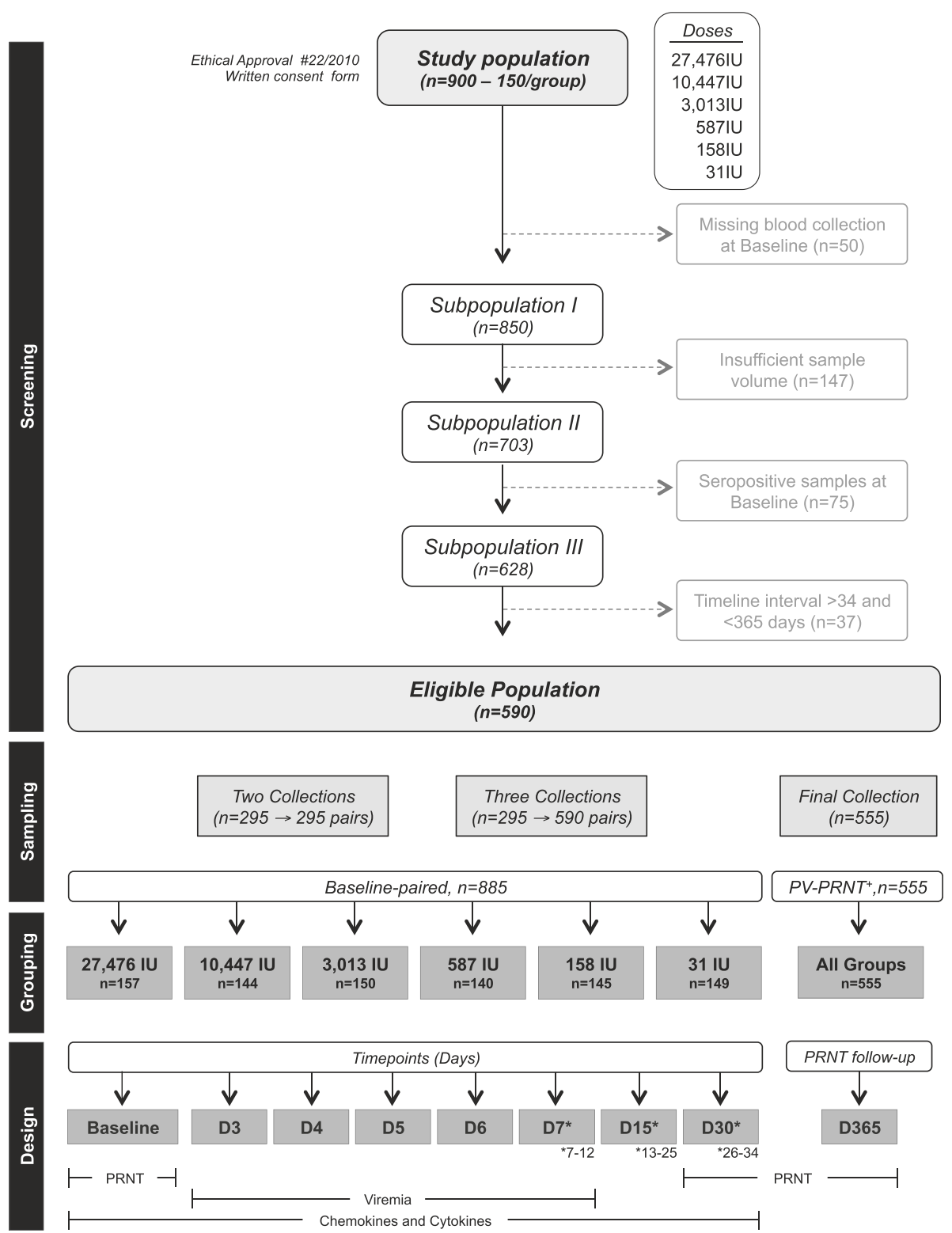

Figure 1 Flowchart illustrating the study population and experimental design. The study was organized in four phases: Screening, Sampling, Grouping and Design. "Screening" of volunteers was performed taking into account: i) missing blood collection at baseline $(n=50)$; ii) insufficient sample volume ( $n=147)$; iii) seropositivity at baseline $(n=75)$ and iv) timeline interval of blood collection $>34$ days and $<365$ days after primary vaccination $(n=37)$. The eligible population comprises 590 primovaccinees. "Sampling" consisted of either two $(n=295)$ or three $(n=295)$ blood collections. The serum samples were paired to their respective sample collected at baseline, resulting in a total of 885 baseline-paired samples. "Grouping" was performed according to the dose of 17DD YF vaccine administered (27,476 IU; 10,447 IU; 3,013 IU; 587 IU; $158 \mathrm{IU}$ and 31 IU). Paired samples were further subcategorized according and the timepoint (days) in which the sample was collected (Baseline; D3; D4; D5; D5; D7 (7-12); D15 (13-25) and D30(26-34). A final blood collection of these volunteers was taken at 365 days after primary vaccination $(n=555)$ to monitor anti-YF antibody status. "Design" included: i) PRNT assays performed at baseline, D30 and D365; ii) Viremia quantified at D3, D4, D5, D6 and D7 and iii) Kinetics of serum chemokines and cytokines evaluated from baseline to D30.

D5, D6 and D7. Chemokines and cytokines were evaluated at Baseline, D3, D4, D5, D6, D7, D15 and D30. The volunteers who showed negative PRNT results at D30 $(n=35)$ were revaccinated with the current vaccine. PRNT follow-up of those volunteers with positive PRNT results after primary vaccination $\left(\mathrm{PV}^{\mathrm{PRNT}}{ }^{+}\right.$, $\mathrm{n}=555)$ was performed in a final blood sample collected at endpoint (D365).

\section{Neutralizing antibody test - PRNT}

The antibody titer against Yellow Fever virus was defined by the Plaque Reduction Neutralization Test 
(PRNT) performed at Virological Technology Laboratory of Bio-Manguinhos (LATEV, FIOCRUZ). This test was conducted as previously described $[9,11]$. Briefly, sera samples from the participants were separated, inactivated and prepared as 2 -fold dilution starting at 1:5, in volumes of $50 \mu \mathrm{l}$ of samples in flat-bottom 96-well tissue culture plates. Twenty-five plaque-forming units (PFU) of yellow fever virus (strain 17D 213/77, lot UEXVFB01, Dec 1011 ) in $50 \mu$ l were added to all wells. A positive serum sample with anti-Yellow Fever virus antibody, properly calibrated by a WHO International Reference Preparation agency, was included in each set of the test. Using linear regression, the $\log _{10}$ dilution of the test and standard serum that reduced the plaque numbers in $50 \%$ relative to the virus control was determined. The mean antibody titer at the $50 \%$ end-point of the standard serum is then calculated and added to the $\log _{10}$ end-point for each sample in order to obtain the values in $\log _{10} \mathrm{mIU} / \mathrm{ml}$. Results are presented in $\log _{10} \mathrm{mIU} /$ $\mathrm{mL}$. The $2.7 \log _{10} \mathrm{mIU} / \mathrm{mL}$ cut-off point was applied to segregate seropositive from seronegative samples as described previously [9].

\section{Viremia}

The RNA of Human serum samples from vaccinated patients with Yellow Fever vaccine was extracted using QIAamp ${ }^{\circ}$ Viral RNA Mini kit (QIAGEN ${ }^{\oplus}$ ) according to the manufacturer's recommendations. Reverse transcription reaction was performed with random primers (F: 5' GCACGGATGTAACAGACTGAAGA3' and R: 5'CCAG GCCGAACCTGTCAT $3^{\prime}$ ) in $20 \mu \mathrm{L}$ of the extracted RNA added to $20 \mu \mathrm{L}$ of High-Capacity cDNA Reverse Transcription mix (Applied Biosystems ${ }^{\circ}$ ). The qRT-PCR assays were performed in the ABIPrism 7500 (Applied Biosystems ${ }^{\circ}$ ) using the probe Fam-CGACTGTGTGGTCCGGCCCA TC-Tamra, directed to the NS5 region of the yellow fever virus $[12,13]$. For constructing the standard curves, the 83 bp fragments from the NS5 viral region obtained by PCR [12] was cloned into a TOPO TA Cloning vector, according to the manufacturer's instructions (Invitrogen ${ }^{\odot}$ ). Serial dilutions from $10^{7}$ to $10^{2}$ plasmid copies per reaction were used to generate the calibration curves for the qRT-PCR assays. The lowest detected concentration was established in 25 copies/reaction.

\section{Chemokine and cytokine quantitation by flow cytometry - CBA}

To assess the levels of chemokines - IL-8/CXCL-8, MCP1/CCL-2, MIG/CXCL-9, IP-10/CXCL-10 and cytokines TNF, INF- $\gamma$, IL-2, IL-4, IL-5 and IL-10 - in serum samples from 17DD-YF vaccinees, Cytometric Bead Array kits (BD Biosciences, California, USA) were used according to manufacturer's protocol and adapted as described previously [14]. Analysis of raw data was performed using the FlowJo cytometry analysis software (FlowJo, Stanford, USA) and the mean fluorescence intensity (MFI) of each bead cluster was evaluated to calculate the each cytokine concentration in the sera of patients. Cytokines concentrations were extrapolated according to the standard curve created by serial dilutions of the positive control. The final data for the kinetics timeline design were expressed as fold changes based on the baseline concentration for all chemokines and cytokines tested.

\section{Data analysis}

The overall kinetics profile of viremia, chemokine and cytokines elicited by 17DD-YF vaccine subdoses were evaluated in comparison to the current dose (27,476 IU) as standard. Equivalence between current dose and subdoses was considered when the median fold change at a given timepoint did not differ as compared to the current dose (Man-Whitney test). The peaks of fold changes along the timeline were also taken into account as a relevant feature for equivalence assessment and highlighted by *. The analyses of PRNT titers were performed in a categorical fashion using the cut-off point of $2.7 \log _{10} \mathrm{IU} / \mathrm{mL}$ as a threshold to segregate positive from negative results. Gray-colored rectangles were used to highlight equivalence in the kinetics profiles. The Prism GraphPad Software version 5.0 (San Diego, CA, USA) and Microsoft Office Excel 2010 were used for kinetics timeline graphic arts and data mining.

\section{Results}

A fifty-fold lower dose of 17DD-YF vaccine is able to trigger similar immunogenicity as evidenced by significant titers of anti-yellow fever virus neutralizing antibodies

The current dose of Yellow Fever vaccine distributed by Bio-Manguinhos is 27,476 IU, which is approximately seven times higher than the dose recommended by WHO. Therefore, in order to investigate the immunogenicity of lower doses of the 17DD Yellow Fever vaccine, a dose-sparing study was designed and performed including six doses of 17DD-YF vaccine as shown in Figure 1.

Figure 2A shows the results on the Plaque Reduction Neutralization Test (PRNT) in sera samples of volunteers vaccinated by several doses of 17DD Yellow Fever vaccine. The threshold of $2.70 \log _{10} \mathrm{mIU} / \mathrm{mL}$ was used as a cut-off to segregate seropositive from seronegative samples. The results show that seropositivity was high among vaccinees of all doses with percentages above 97\% of seroconversion down to dose 587 IU of YF vaccine. At doses $158 \mathrm{IU}$ and $31 \mathrm{IU}$, the percentage of seroconversion was $89 \%$ and $57 \%$, respectively.

Additional analysis of PRNT at D365 indicated that only the lower dose (31 IU) was associated with significant seroreversion. In fact, only $89 \%$ of primary 

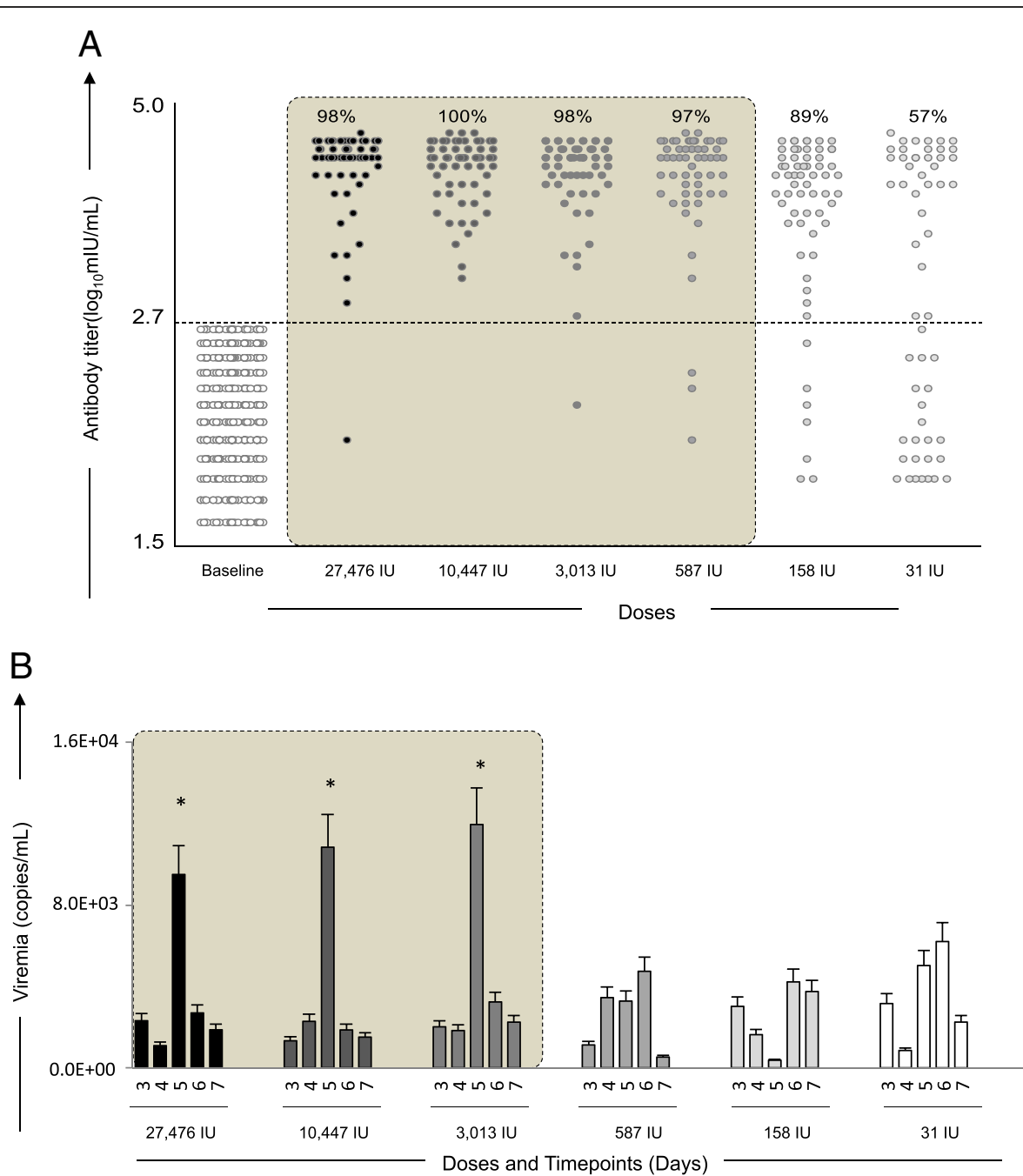

Figure 2 Immunogenicity and Viremia kinetics following 17DD-YF primary vaccination with different doses (27,476 IU-current; 10,447 IU; 3,013 IU; 587 IU; 158 IU and 31 IU). (A) Anti-YF neutralizing antibody titers ( $•=$ current dose and fades for subdoses) were measured by PRNT assay carried out 26-34 days (D30) after primary vaccination, as described in Methods. PRNT antibody titers are expressed in log $10 \mathrm{mIU} / \mathrm{mL}$ and $2.7 \log _{10} \mathrm{mlU} / \mathrm{mL}$ as the cut-off point to segregate seropositive from seronegative samples. Significant seropositivy rates (>95\%) are highlighted by gray rectangle. (B) Viremia $(\mathbf{m}$ =current dose and fades for subdoses) was quantified by Real-time PCR at D3, D4, D5, D6 and D7 after primary vaccination as described in Methods. Viremia results are expressed in copies/mL. Kinetics profiles equivalent to current dose (27,476 IU) are highlighted by gray rectangle. The peaks of fold changes along the timeline were also taken into account as a relevant feature for equivalence assessment and highlighted by *.

vaccinees that received the $31 \mathrm{IU}$ subdose persisted with positive PRNT results one year after primary vaccination (Additional file 1: Figure S1).

\section{A ten-fold lower dose of 17DD-YF vaccine elicits equivalent viremia kinetics with an early peak at five days after primary vaccination}

Figure $2 \mathrm{~B}$ shows the kinetics timeline of Viremia following 17DD-YF primary vaccination with the dose currently used and subdoses. It is possible to observe a significant viremia peak at D5 after primary vaccination with the doses 27,476 IU, 10,447 IU and 3,013 IU. The kinectics of viremia at the lower doses is distinct, with a late and lower-magnitude peak at D6 post-vaccination.

A Fifty-fold lower dose of 17DD YF vaccine is able to trigger equivalent kinetics of IL-8/CXCL-8 and MCP-1/ CCL-2 as observed for PRNT seropositivity.

Figure 3 shows the results of IL-8/CXCL- 8 and MCP$1 / \mathrm{CCL}-2$ kinetics in sera samples from Yellow Fever vaccinees. The chemokine results are displayed as Baseline fold changes for each timepoint. Data analysis demonstrates that doses as low as $587 \mathrm{IU}$ are able to induce similar kinetics for IL-8/CXCL-8 and MCP-1/ CCL-2, characterized by peaks at D3 or D6, and D5 respectively. 


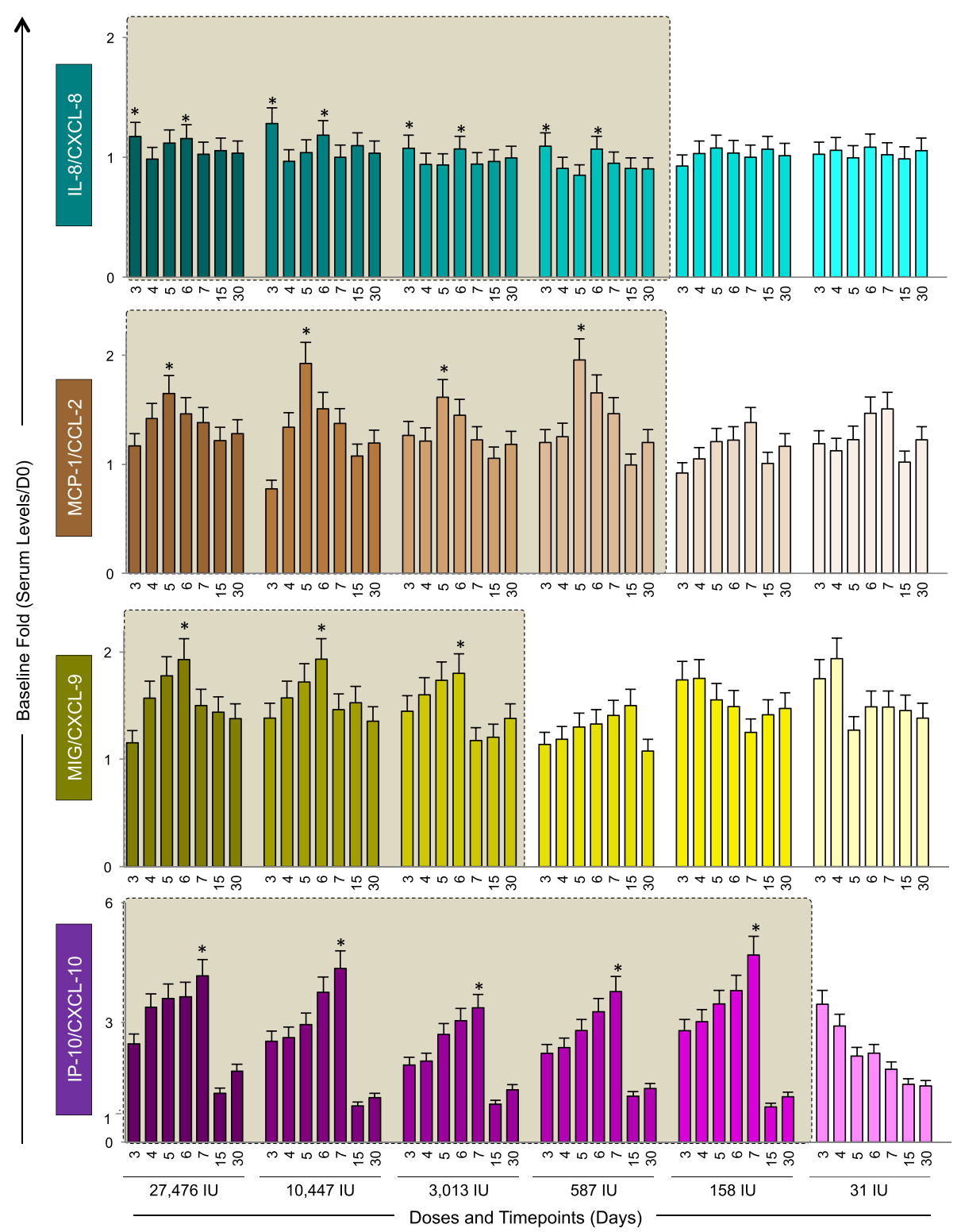

Figure 3 Kinetics of serum chemokines following 17DD-YF primary vaccination with different doses $(27,476$ IU-current; 10,447 IU;

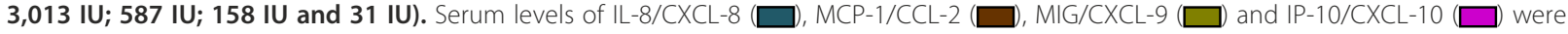
measured by cytometric beads array (CBA) as described in Methods. The colors assigned for the current dose fade away for subdoses. Results are expressed as baseline fold change of each timepoint (Serum level/D0). Kinetics profiles equivalent to current dose (27,476 IU) are highlighted by gray rectangle. The peaks of fold changes along the timeline were also taken into account as a relevant feature for equivalence assessment and highlighted by *.

The MIG/CXCL-9 kinetics induced by a ten-fold downscaled dose resembles the current dose as observed for the viremia kinetics

Figure 3 also shows the results of MIG/CCL-9 and IP-10/ CXCL-10 kinetics in sera samples from Yellow Fever vaccinees. For the MIG/CCL-9, similar increasing kinetics with peak at D6 was observed upon vaccination with doses as low as 3,013 IU specifically one day after the viremia peak (Figures 2 and 3, respectively). The chemokine
IP-10/CXCL-10 displayed the highest baseline fold change magnitude (up to 6 times) with peak of production at D7, except at the lowest dose of vaccine (31 IU), which showed an inverted kinetics profile.

The kinetics of pro-inflammatory cytokines (TNF, IFN- $\gamma$ and IL-2) progresses similarly down to a ten-fold lower dose of 17DD-YF vaccine.

Figure 4 displays the results of the baseline fold changes in the serum pro-inflammatory cytokines TNF, 


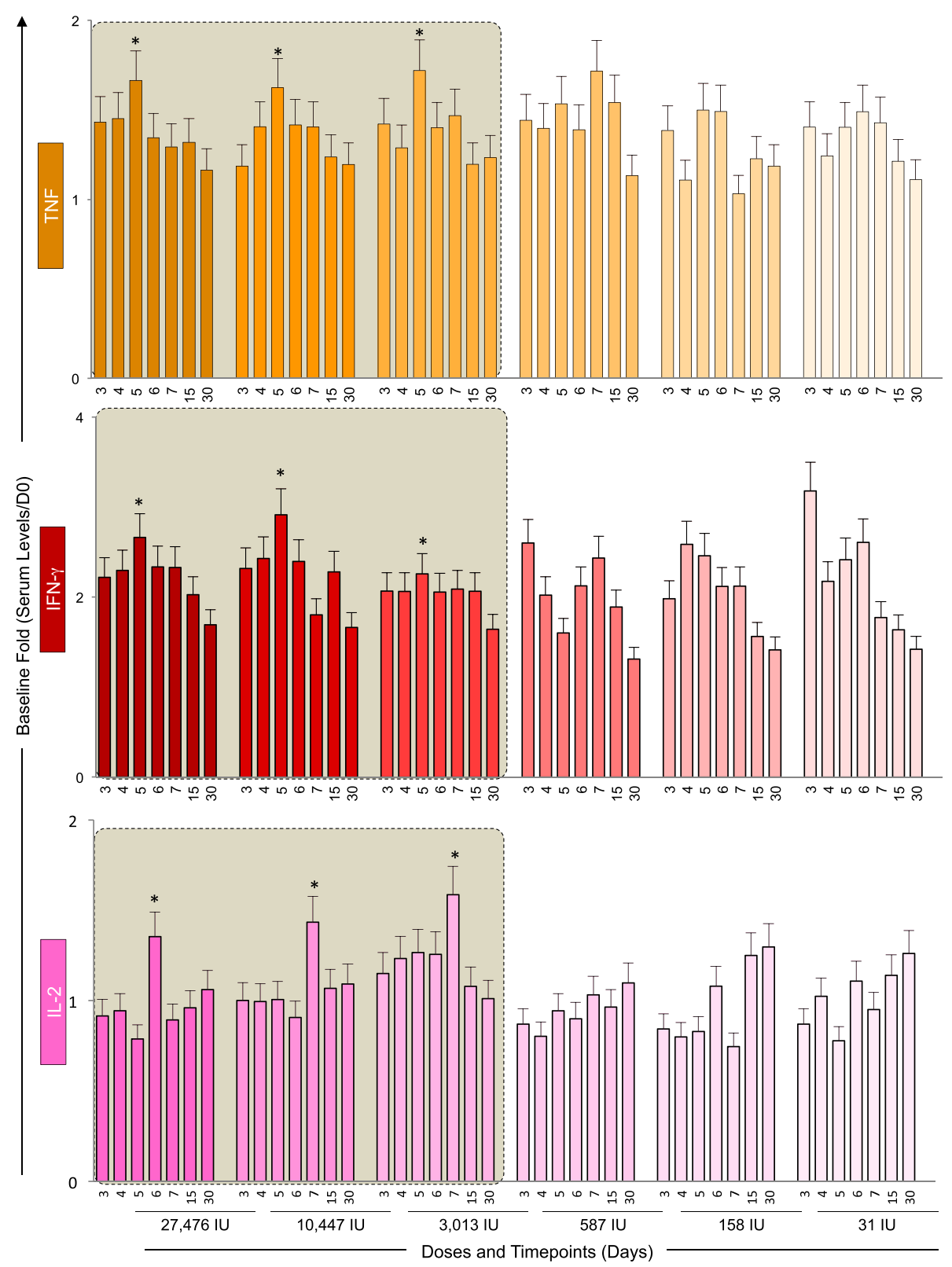

Figure 4 Kinetics of pro-inflammatory cytokines following 17DD-YF primary vaccination with different doses (27,476 IU-current; 10,447 IU; 3,013 IU; 587 IU; 158 IU and 31 IU). Serum levels of the pro-inflammatory cytokines TNF ( $\square$ ), IFN-ץ $\square$ ) and IL-2 ( $\square$ ) were measured by cytometric beads array (CBA) as described in Methods. The colors assigned for the current dose fade away for subdoses. Results are expressed as baseline fold change of each timepoint (Serum level/D0). Kinetics profiles equivalent to current dose (27,476 IU) are highlighted by gray rectangle. The peaks of fold changes along the timeline were also taken into account as a relevant feature for equivalence assessment and highlighted by *

IFN- $\gamma$ and IL-2. The kinetics of these cytokines demonstrates similar peak of TNF and IFN- $\gamma$ at D5 after vaccination with the doses $27,476 \mathrm{IU}, 10,447 \mathrm{IU}$ and $3,013 \mathrm{IU}$ in agreement with the peak of viremia. IL-2 production display peak approximately one day after TNF and IFN- $\gamma$ (D6 or D7) for the these three higher doses. Conversely, the IL-2 kinetics induced by the three lowest doses (587IU, 158IU and 31IU) is characterized by a shift of IL-2 peak towards D30.
Peaks and troughs of IL-10 serum levels upon vaccination with 17DD YF vaccine - a distinguished modulatory cytokine kinectics

Figure 5 demonstrates the results of baseline fold changes for the regulatory cytokines (IL-4, IL-5 and IL-10). The IL-4 secretion pattern was very similar throughout the subdoses, with late peak at Day 30 regardless of the vaccine dose given. For IL-5 and IL-10, similar kinetics profiles were observed to doses as low 


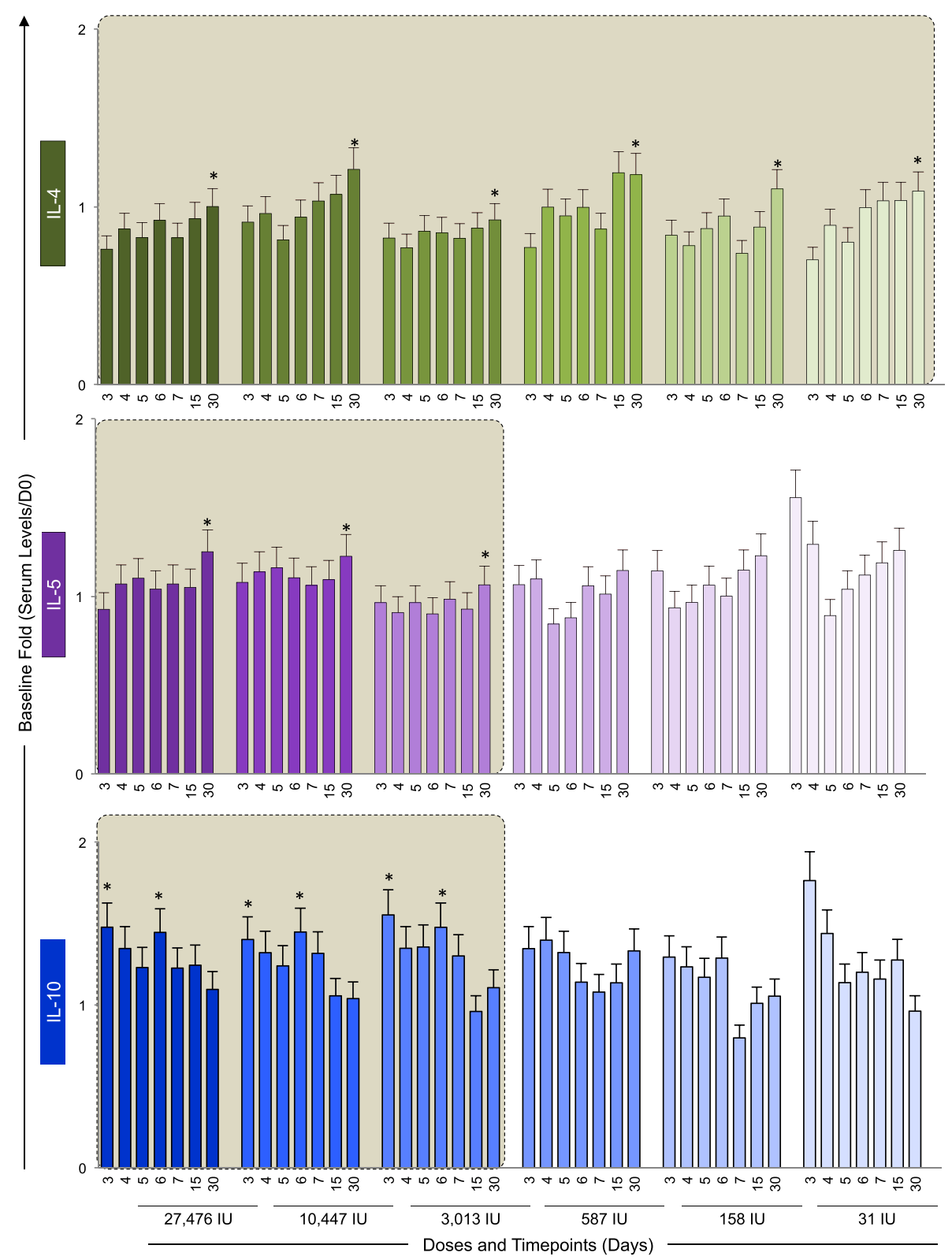

Figure 5 Kinetics of regulatory cytokines following 17DD-YF primary vaccination with different doses $(27,476$ IU-current; 10,447 IU; 3,013 IU; 587 IU; 158 IU and $\mathbf{3 1}$ IU). Serum levels of the regulatory cytokines IL-4 ( $\square$, IL-5 ( $\square$ ) and IL-10 $\square$ ) were measured by cytometric beads array (CBA) as described in Methods. The colors assigned for the current dose fade away for subdoses. Results are expressed as baseline fold change of each timepoint (Serum level/D0). Kinetics profiles equivalent to current dose (27,476 IU) are highlighted by gray rectangle. The peaks of fold changes along the timeline were also taken into account as a relevant feature for equivalence assessment and highlighted by *.

as 3,013 IU. However, IL-10 production presented a distinguished modulatory cytokine kinectics with early peak at day 3 with subsequent trough after day 4 or day 5 that upsurges at day 6 , whereas IL- 5 peaks at day 30 as observed for IL-4. Lower doses of the vaccine do not present the specific IL-10 peak and through kinetics, but rather present inverted pattern with increased IL-10 production in either earlier or later timepoints.
IFN- $\gamma$ and IL-10 are relevant complementary biomarkers that in addition to PRNT and viremia support a safe use of ten-fold lower subdose of 17DD YF vaccine

The overall analysis of serum chemokine and cytokines in addition to PRNT and viremia allowed the identification that six out of ten biomarkers (MIG/CXCL-9, TNF, IFN- $\gamma$, IL-2, IL-5 and IL-10) display equivalent kinetics timeline with viremia and PRNT (Figure 6A). On the 

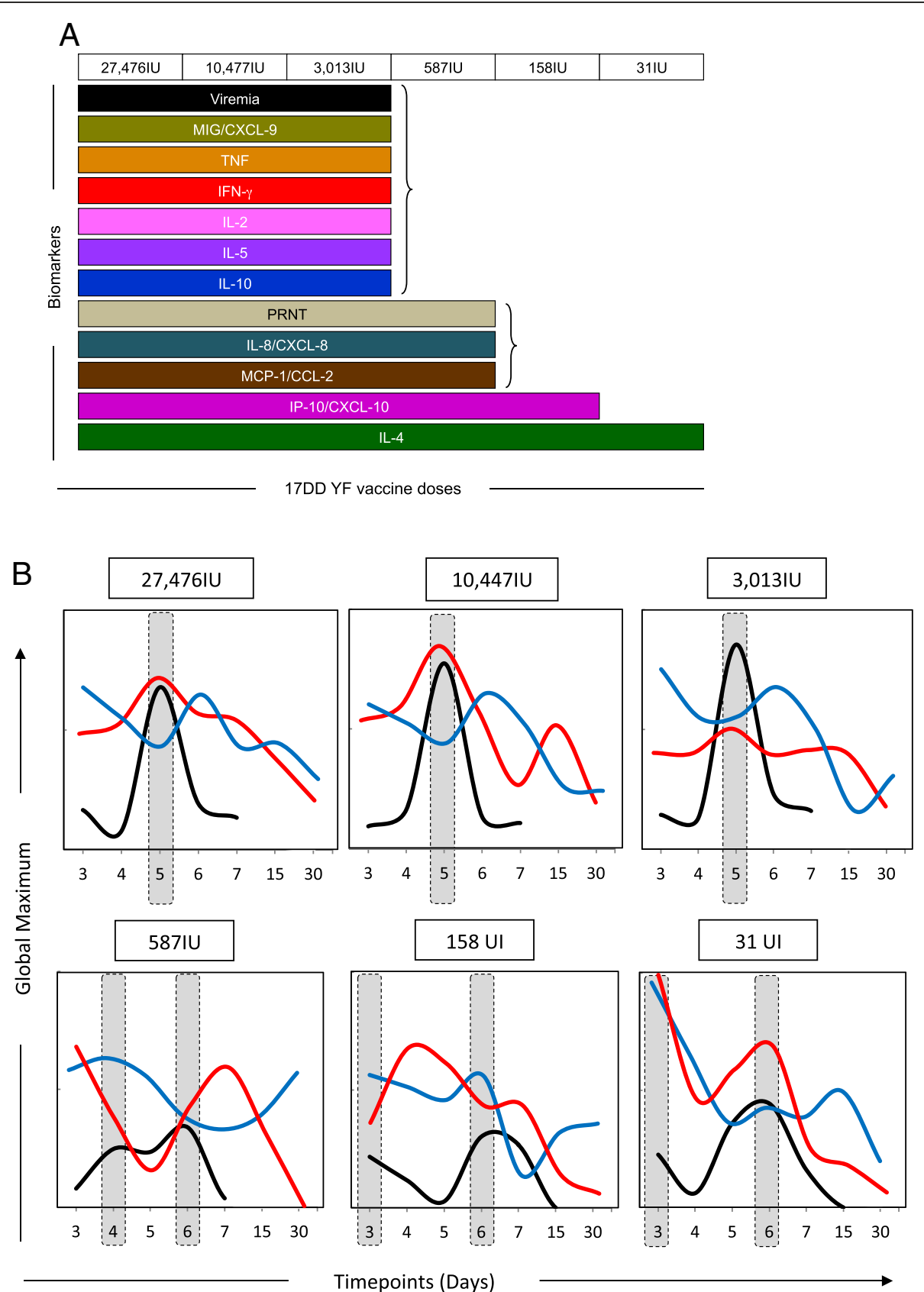

Figure 6 Snapshot of immunological and virological biomarkers following 17DD-YF primary vaccination with different doses (27,476 IU-current; 10,447 IU; 3,013 IU; 587 IU; 158 IU and 31 IU). (A) Selection of biomarkers with equivalent kinetics timeline with viremia

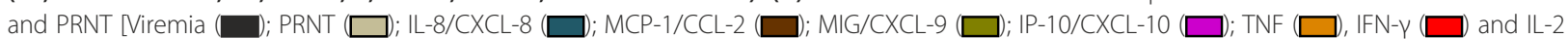

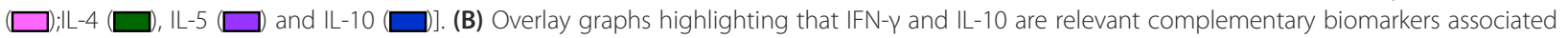
with the viremia kinetics. Results are expressed as the global maximum values of each timepoint to baseline (viremia, $\mathbf{C}$, range 0 to $1.6 \mathrm{E}+04$ copies/mL; INF- $\gamma$, —, range 0 to 4 baseline fold; IL-10, - , range 0 to 2 baseline fold).

other hand, biomarkers (IL-8/CXCL-8 and MCP-1/CCL2) were demonstrated as similar in kinetics with the PRNT positivity. Two biomarkers (IP-10/CXCL-10 and IL-4) did not display any similarity with PRNT and viremia, the current conventional correlates of protection (Figure 6A).
It is important to highlight that IFN- $\gamma$ and IL-10 are relevant complementary biomarkers associated with the viremia kinetics. Together, these biomarkers in addition to PRNT and viremia could support the safe use of ten-fold lower subdose (3,013 IU) of 17DD YF vaccine (Figure 6B). The 3,013 IU subdose induced 
IL-10 trough simultaneously to viremia and IFN- $\gamma$ peaks at D5, similar to the dose currently used (Figure 6B).

\section{Discussion}

In the present study, individuals vaccinated with subdoses of 17DD vaccine were tested for several molecules such as cytokines, chemokines as well as the viral load and the neutralizing antibody titers in a time and dosedependent fashion. The kinectics of these molecules were thoroughly investigated taking into consideration several vaccine doses and immune biomarkers of effective vaccination were proposed.

The results demonstrated that a fifty-fold lower dose of 17DD-YF vaccine (587 IU) is able to trigger similar immunogenicity as evidenced by significant titers of anti-YF PRNT. However, it is important to consider whether the load of antigen exposure during primary vaccination would affect immunogenicity. Only subdoses as low as 3,013 IU elicit viremia kinetics with an early peak at five days after primary vaccination equivalent to the current dose (27,476 IU), while other subdoses show a distinct, lower in magnitude and later peak at day 6 post-vaccination. It is well described that an optimal antigen exposure is necessary for the generation of protective response. In the context of live-attenuated virus, lower doses of vaccine should be able to elicit ideal immunogenicity since the virus replicates within the organism and amplifies its antigenic exposure. This fact indicates that viremia results should be taken together with PRNT titers on the decision making for dose sparing $[9,10,15]$.

In addition to that, although subdoses as low as $587 \mathrm{IU}$ is able to trigger equivalent kinetics of IL-8/ CXCL- 8 and MCP-1/CCL-2, only subdoses downscaled to 3,013 IU are able to trigger similar kinetics of MIG/ CXCL-9, pro-inflammatory (TNF, IFN- $\gamma$ and IL-2) and modulatory cytokines (IL-5 and IL-10).

It is important to note that our results indicate that even subdoses of 17DD yellow fever vaccine have the proper amount of YF vaccine virus to compensate for the possible loss during transportation of the vaccine or when the cold chain is not secured. In addition, the vaccine formulation is comprised of sorbitol and gelatin, which are used as vaccine stabilizers, and the vaccine preparation is lyophilized, allowing for more stability of the vaccine formulation.

Previous studies have demonstrated that the balance of cytokines is crucial to generate a pro-inflammatory response upon vaccination [16-22]. In this regard, TNF$\alpha$ is an important molecule in this scenario. TNF- $\alpha$ produced by neutrophils and monocytes upon antigenic stimuli seem to play a role in inducing an initial proinflammatory response in either primo-vaccination or upon re-vaccination [22]. The levels of this cytokine also correlate with the PRNT titers, which indicates that the production of TNF- $\alpha$ may be important to generate an immunological environment for production of neutralizing antibodies [22].

Conversely, the lack of seroconversion after YF-17DD primary vaccination promotes a regulatory status upon antigen recall, with lower synthesis of TNF- $\alpha$ by neutrophils and monocytes. In addition, the absent production of neutralizing antibodies correlates with enhanced synthesis of modulatory cytokines such as IL-10 produced by $\mathrm{CD}^{+} \mathrm{T}$ cells compared with all other groups [22].

These results are in agreement with the present findings that indicate a peaks and troughs in IL-10 levels that are opposite to viremia peaks and peak levels of TNF- $\alpha$ and IFN- $\gamma$. The trough of IL-10 levels at day 5 may be associated with retreated modulatory response during peak viremia, which allows for the development and maturation of antigen presenting cells (APCs) as well as higher expression of $\mathrm{MHC}$ in the surface of these APCs [19]. The assembly of neutralizing antibodies is dependent upon antigenic presentation, mediated by MHC-II presentation by APCs, which endorses the data associating lower IL-10 synthesis, and higher TNF- $\alpha$ and IFN- $\gamma$ production with PRNT titers and Viral load (Figure 6). The late IL-2 production may be associated with proliferation and clonal expansion of memory $\mathrm{CD}^{+}$ and $\mathrm{CD}^{+} \mathrm{T}$ cells [21].

The kinetics observed in this study was very similar to the one found in previous study demonstrating early and strong cytokine production on 5-7 days after vaccination [18]. The overall cytokine secretion kinetics of 17DD YF vaccinees showed a transient peak of proinflammatory molecules and viral load along with trough of IL-10 secretion at day 5, which draws back toward a mixed/regulatory pattern at later times points in day 15 and day 30. A robust early response is important for maintaining a protective response as indicated by neutralizing antibodies.

Regarding the standardization of the serum biomarkers, we believe that the measuring of viremia, cytokines and chemokines could be easily standardized for regular use. Our group has advanced in this respect as previously described [14]. In addition, Chao et al. [13] and Jonsson et al. [23] have already described the correlation between the quantitation of YFV by real-time PCR and TCID50 or plaque assay lysis, which indicates that viral load could be implemented as a test with high likelihood to plaque assay lysis [13,23].

All in all, the results indicate that subdoses of 17DD vaccines are able to elicit neutralizing antibodies, peak viremia and strong pro-inflammatory response in a timeline similar to the one observed with a higher dose in current used. 


\section{Conclusions}

The analysis of serum biomarkers IFN- $\gamma$ and IL-10, in association to PRNT and viremia, support the recommendation of use of a ten-fold lower subdose (3,013 IU) of 17DD-YF vaccine.

\section{Additional file}

Additional file 1: Figure S1. Persistence of Anti-YF neutralizing antibody titers one year after 17DD-YF primary vaccination with different doses (27,476 IU-current; 10,447 IU; 3,013 IU; 587 IU; 158 IU and 31 IU). PRNT assay was carried out 365 days (D365) after primary vaccination, as described in Methods. PRNT antibody titers $(\cdot=$ current dose and fades for subdoses) are expressed in $\log _{10} \mathrm{mlU} / \mathrm{mL}$ and $2.7 \log _{10} \mathrm{mlU} / \mathrm{mL}$ as the cut-off point to segregate seropositive from seronegative samples. Significant persistence of seropositivity (>95\%) as compared to D30 is highlighted by gray rectangle.

\section{Abbreviations}

APCs: Antigen presenting cells; CCL-9: Chemokine receptor type nine; CCL-2: Chemokine receptor type two; CD4: Cluster of differentiation four; CD8: Cluster of differentiation eight; CNPq: Conselho Nacional de Desenvolvimento Científico e Tecnológico; CPqRR: Centro de Pesquisas René Rachou; CXCL-8: C-X-C Chemokine receptor type eight; CXCL-9: C-X-C Chemokine receptor type nine; $\mathrm{CXCL}-10$ : $\mathrm{C}-\mathrm{X}-\mathrm{C}$ Chemokine receptor type ten; CXCL-3: C-X-C Chemokine receptor type tree; D3: Day 3; D4: Day 4; D5: Day 5; D6: Day 6; D7: Day 7; D15: Day 15; D30: Day 30; FAPEMIG: Fundação de Amparo à Pesquisa do estado de Minas Gerais; FIOCRUZ: Fundação Oswaldo Cruz; IFN- ү: Interferon gamma; IL-8: Interleukin eight; IL-5: Interleukin five; IL-4: Interleukin four; IL-10: Interleukin ten; IL-2: Interleukin two; IL-10: Interleukin 10; IP-10: Interferon gamma-induced protein ten; IU: International unit; LATEV: Laboratório de Tecnologia Virológica de Bio-Manguinhos;

$\log _{10}$ : Logaritmo ten; MCP-1: Monocyte chemotactic protein-1; MFI: Mean fluorescence intensity; MHC: Major histocompatibility complex; MIG: Monokine induced by gamma interferon; mIU/ml: Milli- international units per milliliter; MIP-1 $\beta$ : Macrophage inflammatory protein 1 beta; $n$ : Number; PCR: Polymerase chain reaction; PDTIS: Rede de Plataformas Tecnológicas do Programa de Desenvolvimento Tecnológico em Insumos para Saúde; PFU: Forming unit board; PNI: Programa Nacional de Imunização; PRNT: Plaque reduction neutralization test; PROEP: Programa de Expansão da Educação Profissional; PV: Primary vaccination; RNA: Ribonucleic acid; SVS: Secretariat of health surveillance; TNF- a: Tumor necrosis factors alpha; WHO: World health organization; YF: Yellow fever; YF NS5: Region five of yellow fever virus.

\section{Competing interests}

The authors declare that they have no competing interests.

\section{Authors' contributions}

Thirteen authors (MLSM, RHGF, MSF, RG, AMYY, LFCA, SMBL, GRSS, DAH, RC, MLFL, AH and RMM) are employees at the 17DD-YF vaccine manufacturer (Bio-Manguinhos, Fundação Oswaldo Cruz), and five authors work in other units of Fundação Oswaldo Cruz (VPM, LABC, RMRN, ATC and OAMF). Bias from competing interest was prevented by: (1) collaboration of two general clinical physicians (RAVF and EPF) from Instituto de Biologia do Exército; Rio de Janeiro, Brazil, with experience in infectious diseases. RAVF and EPF contributed with critical overview of the study design, volunteers' immunization and medical care, blood sample collection, and supervision of data interpretation. The Collaborative Group for the Study of Yellow Fever Vaccines was responsible for carrying out the study and (2) inclusion of two independent university professionals working as Undergraduate students (PAE and GVR) or Post-doctoral researchers (ACCA and JGCR) in the field of infectious diseases, responsible for blind sample handling and processing, data collection, and statistical analysis. All authors have read and approved the final manuscript.

\section{Acknowledgements}

We thank the Program for Technological Development of Health Products (PDTIS) for technological development in tools for health; the Oswaldo Cruz Foundation (FIOCRUZ) for use of its facilities; the National Council for
Scientific and Technological Development (CNPq); Minas Gerais State Research Foundation (FAPEMIG) and Brazil's Ministry of Health.

\section{Financial support}

Fundação de Amparo à Pesquisa do Estado de Minas Gerais (FAPEMIG), Bio-Manguinhos/FIOCRUZ; PROEP/CPqRR/FIOCRUZ; Conselho Nacional de Desenvolvimento Científico e Tecnológico (CNPq), Programa Nacional de Imunizações (PNI), Secretaria de Vigilância em Saúde (SVS).

\section{Author details}

${ }^{1}$ Centro de Pesquisas René Rachou - Fiocruz, Belo Horizonte, Minas Gerais, Brazil. ${ }^{2}$ Bio-Manguinhos, Fiocruz, Rio de Janeiro, Brazil. ${ }^{3}$ Escola Nacional de Saúde Pública, Fiocruz, Rio de Janeiro, Brazil. ${ }^{4}$ Instituto Oswaldo Cruz, Fiocruz, Rio de Janeiro, Brazil. ${ }^{5}$ Instituto de Biologia do Exército, Rio de Janeiro, Brazil.

Received: 7 April 2014 Accepted: 3 July 2014

Published: 15 July 2014

\section{References}

1. Vasconcelos PF: Yellow fever. Rev Soc Bras Med Trop 2003, 36(2):275-293.

2. Tomori O: Yellow fever: the recurring plague. Crit Rev Clin Lab Sci 2004, 41(4):391-427

3. Gubler DJ: The global resurgence of arboviral diseases. Trans $R$ Soc Trop Med Hyg 1996, 90(5):449-451

4. Pulendran B: Learning immunology from the yellow fever vaccine: innate immunity to systems vaccinology. Nat Rev Immunol 2009, 9(10):741-747.

5. (WHO) WHO: Expert Committee on Biological Standardization: Requirements for yellow fever vaccine. In Bolletin Requirements for Biological Substances. Geneve: WHO; 2008.

6. (WHO) WHO: Searchable Database of WHO Pre Qualified Vaccines: WHO 2014. Accessed 10 of July 2014. Date of last revision of vaccine list: 01 April 2014. http://www.who.int/immunization_standards/vaccine_quality/ $P Q \_$vaccine_list_en/en/.

7. Camacho LA: Yellow fever and public health in Brazil. Cad Saude Publica 2008, 24(3):482-483.

8. Freire MS, Mann GF, Marchevsky RS, Yamamura AM, Almeida LF, Jabor AV Malachias JM, Coutinho ES, Galler R: Production of yellow fever 17DD vaccine virus in primary culture of chicken embryo fibroblasts: yields, thermo and genetic stability, attenuation and immunogenicity. Vaccine 2005, 23(19):2501-2512.

9. Martins RM, Maia Mde L, Farias RH, Camacho LA, Freire MS, Galler R, Yamamura AM, Almeida LF, Lima SM, Nogueira RM, Sá GR, Hokama DA, de Carvalho R, Freire RA, Pereira Filho E, Leal Mda L, Homma A: 17DD yellow fever vaccine: a double blind, randomized clinical trial of immunogenicity and safety on a dose-response study. Hum Vaccin Immunother 2013, 9(4):879-888.

10. Lopes Ode S, Guimaraes SS, de Carvalho R: Studies on yellow fever vaccine. III-dose response in volunteers. J Biol Stand 1988, 16(2):77-82.

11. Stefano I, Sato HK, Pannuti CS, Omoto TM, Mann G, Freire MS, Yamamura AM, Vasconcelos PF, Oselka GW, Weckx LW, Salgado MF, Noale LF, Souza $\checkmark A$ : Recent immunization against measles does not interfere with the sero-response to yellow fever vaccine. Vaccine 1999, 17(9-10):1042-1046.

12. Mantel N, Aguirre M, Gulia S, Girerd-Chambaz Y, Colombani S, Moste C, Barban V: Standardized quantitative RT-PCR assays for quantitation of yellow fever and chimeric yellow fever-dengue vaccines. J Virol Methods 2008, 151(1):40-46.

13. Chao DY, Davis BS, Chang GJ: Development of multiplex real-time reverse transcriptase PCR assays for detecting eight medically important flaviviruses in mosquitoes. J Clin Microbio/ 2007, 45(2):584-589.

14. Peruhype-Magalhaes V, Martins-Filho OA, Prata A, Silva Lde A, Rabello A, Teixeira-Carvalho A, Figueiredo RM, Guimaraes-Carvalho SF, Ferrari TC, Van Weyenbergh J, Correa-Oliveira R: Mixed inflammatory/regulatory cytokine profile marked by simultaneous raise of interferon-gamma and interleukin-10 and low frequency of tumour necrosis factor-alpha(+) monocytes are hallmarks of active human visceral Leishmaniasis due to Leishmania chagasi infection. Clin Exp Immunol 2006, 146(1):124-132.

15. Ahmed $R$, Pulendran $B$ : Learning vaccinology from viral infections. J Exp Med 2011, 208(12):2347-2349.

16. Querec T, Bennouna S, Alkan S, Laouar Y, Gorden K, Flavell R, Akira S, Ahmed R, Pulendran B: Yellow fever vaccine YF-17D activates multiple 
dendritic cell subsets via TLR2, 7, 8, and 9 to stimulate polyvalent immunity. J Exp Med 2006, 203(2):413-424.

17. Martins MA, Silva ML, Marciano AP, Peruhype-Magalhaes V, Eloi-Santos SM, Ribeiro j G, Correa-Oliveira R, Homma A, Kroon EG, Teixeira-Carvalho A, Martins-Filho OA: Activation/modulation of adaptive immunity emerges simultaneously after 17DD yellow fever first-time vaccination: is this the key to prevent severe adverse reactions following immunization? Clin Exp Immunol 2007, 148(1):90-100.

18. Silva ML, Martins MA, Espirito-Santo LR, Campi-Azevedo AC, Silveira-Lemos D, Ribeiro JG, Homma A, Kroon EG, Teixeira-Carvalho A, Eloi-Santos SM Martins-Filho OA: Characterization of main cytokine sources from the innate and adaptive immune responses following primary 17DD yellow fever vaccination in adults. Vaccine 2011, 29(3):583-592.

19. Ravindran R, Khan N, Nakaya HI, Li S, Loebbermann J, Maddur MS, Park Y, Jones DP, Chappert P, Davoust J, Weiss DS, Virgin HW, Ron D, Pulendran B. Vaccine activation of the nutrient sensor GCN2 in dendritic cells enhances antigen presentation. Science 2014, 343(6168):313-317.

20. Campi-Azevedo AC, de Araujo-Porto LP, Luiza-Silva M, Batista MA, Martins MA, Sathler-Avelar R, da Silveira-Lemos D, Camacho LA, de Menezes MR, de Lourdes de Sousa Maia M, Farias RH, da Silva Freire M, Galler R, Homma A, Ribeiro JG, Lemos JA, Auxiliadora-Martins M, Caldas IR, Elói-Santos SM, Teixeira-Carvalho A, Martins-Filho OA: 17DD and 17D-213/77 yellow fever substrains trigger a balanced cytokine profile in primary vaccinated children. PLOS ONE 2012, 7(12):e49828.

21. Akondy RS, Monson ND, Miller JD, Edupuganti S, Teuwen D, Wu H, Quyyumi F, Garg S, Altman JD, Del Rio C, Keyserling HL, Ploss A, Rice CM, Orenstein WA, Mulligan MJ, Ahmed R: The yellow fever virus vaccine induces a broad and polyfunctional human memory $\mathrm{CD}^{+} \mathrm{T}$ cell response. J Immunol 2009, 183(12):7919-7930.

22. Luiza-Silva M, Campi-Azevedo AC, Batista MA, Martins MA, Avelar RS, da Silveira LD, Bastos Camacho LA, de Menezes MR, de Lourdes de Sousa Maia M, Guedes Farias RH, da Silva Freire M, Galler R, Homma A, Leite Ribeiro JG, Campos Lemos JA, Auxiliadora-Martins M, Eloi-Santos SM, Teixeira-Carvalho A, Martins-Filho OA: Cytokine signatures of innate and adaptive immunity in 17DD yellow fever vaccinated children and its association with the level of neutralizing antibody. J Infect Dis 2011, 204(6):873-883.

23. Jonsson N, Gullberg M, Lindberg AM: Real-time polymerase chain reaction as a rapid and efficient alternative to estimation of picornavirus titers by tissue culture infectious dose $50 \%$ or plaque forming units. Microbiol Immunol 2009, 53(3):149-154.

doi:10.1186/1471-2334-14-391

Cite this article as: Campi-Azevedo et al: Subdoses of 17DD yellow fever vaccine elicit equivalent virological/immunological kinetics timeline.

BMC Infectious Diseases 2014 14:391.

\section{Submit your next manuscript to BioMed Central and take full advantage of:}

- Convenient online submission

- Thorough peer review

- No space constraints or color figure charges

- Immediate publication on acceptance

- Inclusion in PubMed, CAS, Scopus and Google Scholar

- Research which is freely available for redistribution 